\title{
Higher urinary cortisol levels associate with increased cardiovascular risk
}

\author{
Andrea V Haas' ${ }^{1}$, Paul N Hopkins ${ }^{2}$, Nancy J Brown ${ }^{3}$, Luminita H Pojoga ${ }^{1}$, Jonathan S Williams ${ }^{1}$, Gail K Adler ${ }^{1}$ and \\ Gordon H Williams ${ }^{1}$
}

${ }^{1}$ Division of Endocrinology, Diabetes and Hypertension, Department of Medicine, Brigham and Women's Hospital, Harvard Medical School, Boston, Massachusetts, USA

${ }^{2}$ Cardiovascular Genetics, Department of Internal Medicine, University of Utah School of Medicine, Salt Lake City, Utah, USA

${ }^{3}$ Vanderbilt University Medical Center, Nashville, Tennessee, USA

Correspondence should be addressed to A V Haas: ahaas2@bwh.harvard.edu

\begin{abstract}
There are conflicting data on whether variations of physiologic cortisol levels associated with cardiovascular risk. We hypothesize that prior discordant findings are related to problems associated with varying sample size, techniques for assessing cardiovascular risk and failure to adequately account for environmental factors. To address these issues, we utilized a large sample size, selected the Framingham risk score to compute cardiovascular risk and performed the study in a highly controlled setting. We had two main objectives: determine whether higher, yet physiologic, cortisol levels associated with increased cardiovascular risk and determine whether caveolin-1 (rs926198) risk allele carriers associated with increased cardiovascular risk. This was a cross-sectional study of 574 non-diabetic individuals who completed a common protocol. Data collection included fasting blood samples, blood pressure measurements and a 24-h urine-free cortisol collection. Five hundred seventeen of these participants also completed caveolin-1 genotyping. Subjects were classified as belonging to either the low-mode or high-mode urine-free cortisol groups, based on the bimodal distribution of urine-free cortisol. In multivariate analysis, Framingham risk score was statistically higher in the high-mode cortisol group (10.22 (mean) \pm 0.43 (s.E.M.)) compared to the low-mode cortisol group $(7.73 \pm 0.34), P<0.001$. Framingham risk score was also statistically higher in the caveolin-1 risk allele carriers $(8.91 \pm 0.37)$ compared to caveolin-1 non-risk allele carriers $(7.59 \pm 0.48)$, $P=0.034$. Overall, the estimated effect on Framingham risk score of carrying the caveolin-1 risk allele was $1.33 \pm 0.61, P=0.029$. Both urinary cortisol and caveolin-1 risk allele status are independent predictors of Framingham risk score.
\end{abstract}

\author{
Key Words \\ - cortisol \\ - cardiovascular health \\ - Framingham risk score \\ - Caveolin-1
}

\section{Introduction}

It is well established that individuals with Cushing's syndrome, a disease of excess glucocorticoid production, have increased rates of hypertension, obesity, type 2 diabetes and cardiovascular (CV) events (1). Likewise, individuals with incidentally discovered adrenal masses and biochemical work-up consistent with subclinical Cushing's syndrome also have increased cardiometabolic risk (2, 3, 4, 5, 6). Even among individuals with biochemical work-up consistent with non-functional adrenal tumors, there is a two-fold elevated risk of diabetes, raising the possibility that 'non-functional' adrenal tumors may actually produce small, yet excess, amounts of glucocorticoids (7). Together, these studies implicate a role of both overt and non-overt hypercortisolism in the pathogenesis of cardiovascular disease (CVD).
Endocrine Connections (2019) 8, 634-640 
However, much less is known about the CV effects of cortisol concentrations within the physiological range. While some studies have shown a link between higher cortisol levels and the presence of CV risk factors $(8,9,10$, $11,12,13)$, others have not $(14,15,16,17)$. The reasons for the observed discordance are uncertain, but could relate to sample size, methods used to assess $\mathrm{CV}$ risk and failure to adequately control for environmental factors. Most prior studies evaluated cortisol's effect on single risk factor analyses (e.g. obesity or glucose intolerance) rather than overall or composite $\mathrm{CV}$ risk. The Framingham risk score is a well validated and widely used prediction tool of 10 -year CV events and death $(18,19)$. To our knowledge, the Framingham risk score has never been utilized to assess the relationship between cortisol and CV risk.

In addition, there is mounting evidence that $\mathrm{CV}$ risk is heritable (20). Members of our group have previously shown that a prevalent caveolin-1 (CAV1) gene variant (rs926198) is related to cardiovascular risk (insulin resistance and the metabolic syndrome) $(21,22)$. CAV1 is a scaffolding protein and is important in CV and kidney tissue signal transduction (22). Accordingly, we sought to determine whether there was a relationship between the CAV1 risk allele and increased cardiovascular risk as well as the possibility of an interaction between cortisol levels, CAV1 gene variants and CV risk $(23,24)$.

To address the potential causes of the disparate findings related to physiologic variations of cortisol concentration and CV risk, we used three novel approaches: (1) a large sample size, (2) tight environmental control and (3) Framingham risk score as the assessment tool for CV risk. The aim of this study was to determine if there is a positive relationship between physiologic cortisol levels and cardiovascular risk. An additional aim was to determine whether CAV1 risk allele carriers associate with CV risk. Accordingly, we hypothesized that both higher urinary cortisol levels as well as caveolin-1 risk allele carriers would correlate with higher Framingham risk scores; those individuals who had both higher cortisol levels and were CAV1 risk allele carriers would have the highest risk.

\section{Methods}

\section{HyperPATH cohort and study protocol}

This was a cross-sectional study from the international Hypertensive Pathotype (HyperPATH) cohort. HyperPATH is an ongoing study to investigate the pathophysiologic mechanisms and genes involved in hypertension and CV disease. All subjects completed a common protocol in clinical research centers located at one of five locations: Brigham and Women's Hospital (Boston, MA, USA), University of Utah Medical Center (Salt Lake City, UT, USA), Vanderbilt University Medical Center (Nashville, TN, USA), Hospital Broussais (Paris, France) and San Salvatore Hospital (Rome, Italy). The protocol was approved by institutional review boards of each site, and informed consent was obtained before participant enrollment.

Details of the full protocol have been described previously $(25,26,27,28)$. In brief, subjects were 18-65 years old and had no known or suspected secondary hypertension, coronary artery disease, stroke, renal insufficiency (serum creatinine $\leq 1.5 \mathrm{mg} / \mathrm{dL}$ ) or psychiatric illness. All antihypertensive medications were discontinued 3-4 weeks prior to laboratory assessment. Subjects completed the protocol after a 7-day isocaloric high-salt diet ( $>200 \mathrm{mEqNa} /$ day). On the final evening of the diet week, subjects were admitted to the Clinical Research Center at their respective study site. Blood pressure and laboratory assessments were obtained in the morning between 08:00 h and 10:00 h while fasting and overnight supine posture. Measurement of urinefree cortisol (UFC) was performed on a single 24-h urine collection. UFC was measured by Coat-A-Count Radioimmunoassay (Diagnostics Products Corp., Los Angeles, CA, USA), with a sensitivity of $0.2 \mu \mathrm{g} / \mathrm{dL}$ and a precision of $4-6.4 \%$. Normal reference range for $24-\mathrm{h}$ UFC by radioimmunoassay is considered $<100 \mu \mathrm{g} / 24 \mathrm{~h}$. For comparison, results of UFC by LC-MS/MS are generally found to be $40 \%$ lower than those with radioimmunoassay (29). Details of additional laboratory assays have been described previously $(13,30)$. Although other results from the HyperPATH cohort have been published, the present analyses are original.

\section{Study end-points}

1. Urinary Free Cortisol: Prior studies have demonstrated that UFC levels in the population are not normally distributed, but rather bimodally distributed $(13,30)$. The bimodal distribution is shifted higher in men than women $(13,30)$. Further, there is evidence of heritability in the higher UFC mode $(13,30)$. Therefore, subjects in this study were classified as either in the low-mode or high-mode cortisol group based on the bimodal distribution of UFC for their respective sex. As previously described by members of our group, the cut-point to determine low-mode vs high-mode was a UFC value below and above $49 \mu \mathrm{g} / 24 \mathrm{~h}$ in women and $59.7 \mu \mathrm{g} / 24 \mathrm{~h}$ in men (30). 
2. Caveolin-1 (CAV1): We used rs926198 as the candidate variant of CAV1. This single-nucleotide polymorphism was selected based on our group's previous analysis of HapMap variants of the CAV1 gene in relation to insulin resistance in the HyperPATH dataset. This variant is associated with insulin resistance, hypertension and the metabolic syndrome $(21,22)$. DNA was genotyped as previously described (21). Individuals were classified as non-risk allele carriers if they were homozygote for the major rs926198 allele and were classified as risk allele carriers if they had one or two alleles for the minor rs926198 allele variant.

3. Framingham Risk Score: The hard coronary heart disease (CHD) 10-year risk calculator was used to determine Framingham risk score as outlined in the Adult Treatment Panel III (18). Points are assigned based on an individual's sex, age, total cholesterol (TC), high-density lipoprotein (HDL) cholesterol, systolic blood pressure (SBP), treatment for hypertension and cigarette smoking. The total risk score sums the points assigned to each risk factor to estimate the 10-year risk of myocardial infarction and/or coronary death (18). All participants with diabetes were excluded given that diabetes is considered a CHD risk-equivalent (18).

\section{Statistical analysis}

Population characteristics for individuals in the lowand high-mode cortisol groups were compared using a Student's t-test. Multivariate regression was used to test the association between urinary cortisol and Framingham risk score. Covariates in the model were selected a priori and were treated as fixed effects. Covariates included site of study and factors that have clinical importance for known CV risk factors, race and BMI. As age, sex, SBP and cholesterol are already incorporated into the Framingham risk score, they were not duplicated as covariates. Participant relatedness (i.e. sibling status) was incorporated into the multivariate model. We also compared low-mode and high-mode subjects using propensity score analyses (STATA PSMATCH2) to ensure that we had assembled study populations balanced on the baseline covariates of race, site of study and BMI. Statistical significance was indicated by $P<0.05$. All statistical analysis was performed using Stata Statistical Software: Release 15 (StataCorp LLC, College Station, TX, USA).

\section{Results}

\section{Baseline characteristics in low- and high-mode cortisol groups}

The HyperPATH cohort included a total of 574 non-diabetic individuals who had a 24-h UFC collection and complete data to calculate a Framingham risk score (Table 1). A total of 433 individuals met criteria for the low-mode urinaryfree cortisol group and 141 individuals for the high-mode urinary-free cortisol group. Individuals in the high mode were on average 2.4 years older $(P=0.03)$ and had a higher mean SBP by $9 \mathrm{mmHg}(P<0.001)$, diastolic blood pressure (DBP) by $5 \mathrm{mmHg}(P<0.001)$, fasting blood glucose by $4 \mathrm{mg} / \mathrm{dL}(P=0.01)$, TC by $14 \mathrm{mg} / \mathrm{dL}(P<0.001)$ and LDL by $14 \mathrm{mg} / \mathrm{dL}(P<0.001)$ than individuals in the low-mode group.

\section{Framingham risk score higher in high-mode cortisol group}

The correlation of individual components of the Framingham risk score with urinary cortisol mode was tested. The low Pearson's coefficient for age (0.10), SBP (0.18), TC (0.15) and HDL (-0.07) indicated the absence of a dominant cardiometabolic risk factor associated with cortisol, further supporting our approach to use the composite Framingham risk score.

On multivariate regression, mean Framingham risk score in the low-mode group was $7.73 \pm 0.34$ (s.E.M.) vs $10.22 \pm 0.43$ in the high-mode group $(P<0.001)$ (Fig. 1). The propensity score coefficient was 2.39 (95\% CI 1.107-3.677), demonstrating adequately balanced groups in regards to the covariates. In the model, higher BMI was independently associated with Framingham risk score. For every increase in BMI of $1 \mathrm{~kg} / \mathrm{m}^{2}$, Framingham risk score increased by 0.37 points. To provide further validation of our findings, we also assessed the association between urinary-free cortisol as a continuous variable and Framingham risk score. As a continuous variable, higher urinary-free cortisol levels associated with higher Framingham risk score: $P=0.01$.

\section{CAV1 minor allele carriers have higher Framingham risk score}

Given the evidence for CV risk heritability and our prior work demonstrating that CAV1 risk allele carriers have greater insulin resistance, we investigated whether

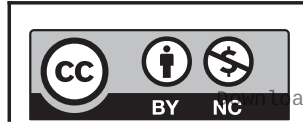

This work is licensed under a Creative Commons Attribution-NonCommercial 4.0 International License. ded from Bioscientifica.com at 04/26/2023 12:05:17AM 
Table 1 Characteristics of study population based on urinary cortisol mode.

\begin{tabular}{l}
\hline \\
\hline Age (years) \\
Sex (female, \%) \\
Race (white \%) \\
BMI (kg/m²) \\
SBP (mmHg) \\
DBP (mmHg) \\
UFC ( $\mu$ g/24 h) \\
Hypertension (\%)* \\
Fasting blood glucose (mg/dL) \\
TC (mg/dL) \\
HDL (mg/dL) \\
LDL (mg/dL) \\
TG (mg/dL) \\
Statin use $(\%)$ \\
Current smoker $(\%)$
\end{tabular}

\begin{tabular}{c}
\hline Low-mode $(n=433)$ \\
\hline $45.3 \pm 10.6$ \\
49.7 \\
88.7 \\
$28 \pm 4$ \\
$136 \pm 24$ \\
$81 \pm 14$ \\
$34 \pm 10$ \\
64.1 \\
$89 \pm 16$ \\
$186 \pm 39$ \\
$45 \pm 19$ \\
$114 \pm 35$ \\
$143 \pm 101$ \\
5.8 \\
10.4
\end{tabular}

\begin{tabular}{c}
\hline High-mode $(n=141)$ \\
\hline $47.7 \pm 8.6$ \\
44.0 \\
89.3 \\
$28 \pm 4$ \\
$145 \pm 20$ \\
$86 \pm 12$ \\
$76 \pm 25$ \\
86.6 \\
$93 \pm 12$ \\
$200 \pm 38$ \\
$42 \pm 13$ \\
$128 \pm 36$ \\
$143 \pm 98$ \\
8.1 \\
9.0
\end{tabular}

\begin{tabular}{c}
\hline $\boldsymbol{\beta}$ \\
\hline 2.4 \\
-5.7 \\
0.6 \\
0 \\
9 \\
5 \\
42 \\
22.5 \\
4 \\
14 \\
-3 \\
14 \\
0 \\
2.3 \\
-1.4 \\
\hline
\end{tabular}

\begin{tabular}{c}
\hline $\boldsymbol{P}$ value \\
\hline 0.03 \\
0.24 \\
0.84 \\
0.55 \\
$<0.001$ \\
$<0.001$ \\
$<0.001$ \\
$<0.001$ \\
0.01 \\
$<0.001$ \\
0.09 \\
$<0.001$ \\
0.98 \\
0.52 \\
0.62
\end{tabular}

Values are represented as means \pm s.D. for continuous variables and percentages for categorical variables. Comparisons across urinary cortisol modes were performed using Student's $t$-test for continuous variables and Fisher exact for binary variables.

*Hypertension was defined as a seated diastolic blood pressure of $\geq 100 \mathrm{mmHg}$ off antihypertensive medications or $\geq 90 \mathrm{mmHg}$ if taking one or more antihypertensive medication.

BMI, body mass index; DBP, diastolic blood pressure; HDL, high-density lipoprotein; LDL, low-density lipoprotein; SBP, systolic blood pressure; TC, total cholesterol; TG, triglycerides; UFC, urinary-free cortisol.

CAV1 risk allele carriers also had higher Framingham risk scores. Of the 574 individuals who had a 24-h UFC as well as complete data to calculate a Framingham risk score, 517 individuals also had DNA analysis for CAV1 genotype. There were 202 subjects who were CAV1 nonrisk allele carriers and 315 subjects who were CAV1 risk allele carriers (247 heterozygotes and 68 homozygotes), and this genotype distribution was in Hardy-Weinberg equilibrium. CAV1 risk allele carriers had a mean \pm s.E.M. Framingham risk score of $8.91 \pm 0.37$ compared to CAV1 non-risk allele carriers of $7.59 \pm 0.48(P=0.03)$ (Fig. 2). As a control, we looked at two other genes in this same population, angiotensinogen and serum/glucose regulated

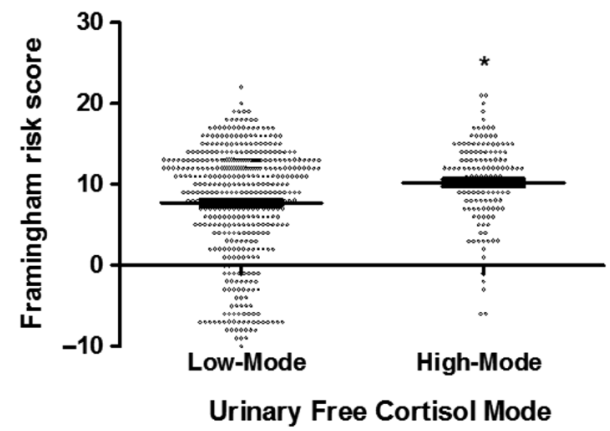

\section{Figure 1}

Framingham risk score by low vs high-mode urinary cortisol. Values represent the mean \pm standard error of the mean (S.E.M.). Mean Framingham risk score in low-mode was $7.73 \pm 0.34$ and in high-mode was $10.22 \pm 0.43$. Multiple linear regression $P<0.001$ adjusted for $\mathrm{BMI}$, study site, race and family relatedness. kinase 1 , which are both associated with salt sensitive hypertension $(31,32)$. We found no relationship between Framingham risk score and angiotensinogen $(P=0.22)$ or serum/glucose regulated kinase 1 risk allele $(P=0.75)$ carrier status.

\section{CAV1, cortisol and Framingham risk score}

CAV1 risk allele carriers were no more likely to be in the low-mode vs high-mode cortisol group $(P=0.37)$. However, given the association of urinary cortisol and CAV1 genotype with Framingham risk score, we tested

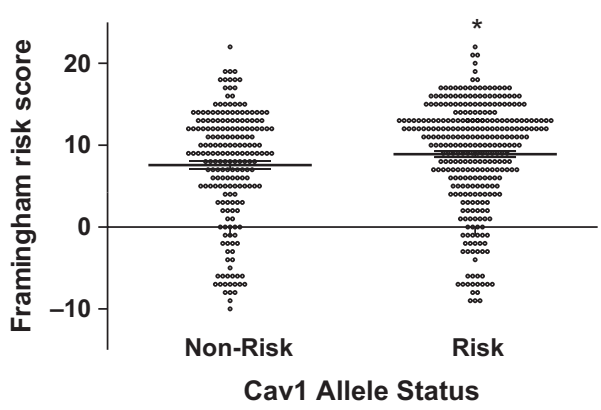

Figure 2

Framingham risk score of Cav1 non-risk vs risk allele (rs926198) carriers. Values represent means \pm S.E.M. Mean Framingham risk score in non-risk allele carriers was $7.59 \pm 0.48$ vs $8.91 \pm 0.37$ in risk allele carriers. Multiple linear regression $P=0.034$ after adjusting for $\mathrm{BMI}$, study site, race and family relatedness.

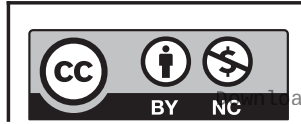

This work is licensed under a Creative Commons Attribution-NonCommercial 4.0 International License. ded from Bioscientifica.com at 04/26/2023 12:05:17AM via free access 
whether the addition of CAV1 risk allele status as a covariate added meaningfully to our multivariate model of Framingham risk score and urinary-free cortisol mode.

In the low-mode UFC group, the presence of a CAV1 risk allele increased Framingham risk score by 1.2 points (non-risk allele carriers $7.15 \pm 0.63$; risk allele carriers $8.38 \pm 0.47)$. In the high-mode UFC group, CAV1 risk allele status mediated an approximate 1.0 point increase in Framingham risk score (non-risk allele carriers 9.78 \pm 0.47 ; risk allele carriers $10.77 \pm 0.66$ ) (Fig. 3). The inclusion of CAV1 risk allele status contributed significantly to the model. Overall, the estimated effect of the risk allele was an increase in Framingham risk score of $1.33 \pm 0.61(P=0.03)$.

\section{Discussion}

Confirming our hypothesis, this study among nearly 600 subjects demonstrated a strong relationship between 24-h UFC levels and predicted CV risk. We have also shown that the CAV1 risk allele was associated with Framingham risk score independently and after incorporation of an individual's 24-h UFC. The lowest Framingham risk scores were those in the low-mode cortisol group and who were CAV1 non-risk allele carriers, while the highest risk scores were those in the high-mode cortisol group and who were CAV1 risk allele carriers, a difference of 3.5 points.

The associations of cortisol and CAV1 on Framingham risk score have meaningful clinical consequences. Even a 1-2 point difference in Framingham risk score can increase a person's 10-year CV risk substantially. For example, men in the low-mode cortisol group had a 10-year CV risk of $4 \%$ compared to men in the high-mode cortisol group, whose risk was $6 \%$ (18). Those in the high-mode cortisol

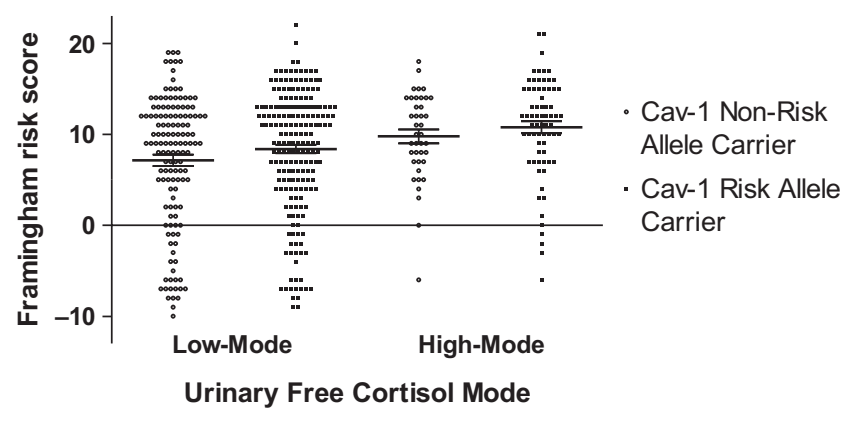

\section{Figure 3}

Framingham risk score in Cav-1 non-risk allele vs risk allele carriers grouped by low and high-mode urinary-free cortisol. Values represent means \pm S.E.M. Mean Framingham risk score in low-mode/non-risk allele carrier was $7.15 \pm 0.63$ vs low-mode/risk allele carrier of $8.38 \pm 0.47$. Mean Framingham risk score in the high-mode/non-risk allele group was $9.78 \pm 0.76$ vs high-mode/risk allele of $10.77 \pm 0.66$. group and CAV1 risk allele carriers had a 2- to 2.5-fold higher 10-year CV risk (8\%) compared to those in the lowmode cortisol group and non-risk allele carriers (3\%).

Our results provide support that there is a positive relationship between physiologic cortisol levels and CV risk. Over 20 prior studies have investigated whether cortisol measurements are related to components of $\mathrm{CV}$ risk. These studies have used various cortisol measurement techniques (morning plasma, salivary, post-dexamethasone suppression, 24-h urine) that assess distinct aspects of the hypothalamic-pituitary-axis. An emerging new technique that may also be of use is measurement of hair cortisol $(33,34)$. However, often the results from different studies have been variable. For example, in a study among 370 men, morning fasting plasma cortisol concentrations were significantly associated with higher blood pressure, plasma glucose concentrations, fasting triglyceride levels and insulin resistance (11). Conversely, in a study among 369 overweight/obese subjects and 160 healthy volunteers, salivary cortisol, 24-h UFC and post-dexamethasone serum cortisol levels did not strongly relate to obesity and the metabolic syndrome (17). The major difficulty with many of these studies is the substantial variability used to control the factors known to modify the reliability of cortisol measurements, and thereby, obscure the ability to identify a positive association. For example, time of day, posture, sleep-wake cycles, eating and stress can modify circulating cortisol levels over a short time interval. The often variable urine collection times and the difference between cortisol's diurnal and creatinine's non-diurnal cycle can adversely affect using 24-h cortisol excretion rates as an index of cortisol production. Also, the potential confounding effects of medications and diet introduce additional heterogeneity in the measurement of cortisol levels. We believe our study improves upon prior research as we had the largest sample size reported to date. In our cohort, the following controls were used: controlled diet, time of day, daily activity and stress factors in a Clinical Research Center setting. The subjects were not taking any medications. Also, we used 24-h cortisol levels as our outcome measurement. Through these techniques, we minimize the potential 'noise' in assessing cortisol production. Finally, we also are the first to use the highly validated Framingham CV risk score calculator as our method of assessment of CV risk, which incorporates age, TC, HDL, SBP, smoking status and sex and we further corrected for BMI and race.

Our findings related to CAV1 risk allele carriers and increased $\mathrm{CV}$ risk are consistent with prior research. Previously, we have shown that the CAV1 risk allele was associated with the metabolic syndrome in both Caucasian

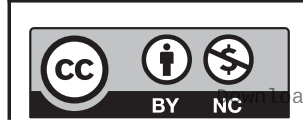

This work is licensed under a Creative Commons Attribution-NonCommercial 4.0 International License. ded from Bioscientifica.com at 04/26/2023 12:05:17AM 
and Hispanic cohorts (21). Building on our prior findings, we have also now demonstrated higher Framingham risk scores for the same CAV1 variant as well as the fact that cortisol and CAV1 are independently associated with CV risk. The mechanism by which cortisol may raise CV risk is not clearly understood. There is evidence implicating a role of glucocorticoids in the modulation of vascular function and reactivity $(35,36)$. Likewise, the pathophysiology of CAV1 in the development of CVD is unknown, but may be related to a role of CAV1 in mineralocorticoid receptor signaling (28).

These findings have importance in potentially identifying novel $\mathrm{CV}$ risk factors and may provide insight into the CVD development. Given the association between physiologic cortisol elevations and CV risk, there may be therapeutic benefit of investigating ways of decreasing cortisol in individuals with greater production. As we move toward personized medicine, the CAV1 risk allele may be more readily available clinically and should alert medical providers of increased CV risk.

Limitations of the study include the cross-sectional design, which prevented us from evaluating a causal relationship between UFC and CV risk. Our findings are highly suggestive but do not prove that physiologically higher cortisol production mediates CV risk. An additional limitation is that we have only one UFC collection for each participant. While our results would be strengthened if we had two UFC collections per participant to account for variability in UFC collections, given that collections were performed under a highly controlled environment, it is anticipated that much of the variability of day-to-day UFC collections would be minimized. We acknowledge that individuals in the high-mode group could have undiagnosed Cushing's or subclinical Cushing's syndrome. However, in the majority of cases, the UFC level was in the high-normal range for the assay. Further, these subjects had no history or clinical evidence of Cushing's syndrome at the time of the study. However, radiographic imaging of the abdomen was not performed to assess for adrenal tumors and neither dexamethasone suppression tests nor late night salivary cortisol were obtained.

\section{Conclusion}

In conclusion, with a large sample size and after controlling for environmental factors, we have shown a positive relationship between physiologic, yet higher, urinary cortisol levels and CV risk as well as CAV1 gene variants and CV risk. As CAV1 risk allele is heritable and high-mode UFC is likely heritable, we add to and further characterize evidence for a heritable component of $\mathrm{CV}$ risk.

\section{Declaration of interest}

The authors declare that there is no conflict of interest that could be perceived as prejudicing the impartiality of the research reported.

\section{Funding}

This work was supported by National Institutes of Health grants T32HL-007609, P50HL055000 (HyperPATH Cohort), UL1RR025758 Harvard Clinical and Translational Science Center, from the National Center for Research Resources; M01-RR02635, Brigham and Women's Hospital, General Clinical Research Center, from the National Center for Research Resources; NIH grants R01HL086907, R01HL11476, R01HL69208, R01HL4765, R01HL59424, R01HL69208, R01HL096518, R01HL104032, K24 HL103845.

\section{References}

1 Pivonello R, Faggiano A, Lombardi G \& Colao A. The metabolic syndrome and cardiovascular risk in Cushing's syndrome. Endocrinology and Metabolism Clinics of North America 200534 327-339, viii. (https://doi.org/10.1016/j.ecl.2005.01.010)

2 Di Dalmazi G, Vicennati V, Garelli S, Casadio E, Rinaldi E, Giampalma E, Mosconi C, Golfieri R, Paccapelo A, Pagotto U, et al. Cardiovascular events and mortality in patients with adrenal incidentalomas that are either non-secreting or associated with intermediate phenotype or subclinical Cushing's syndrome: a 15-year retrospective study. Lancet: Diabetes and Endocrinology 20142 396-405. (https://doi.org/10.1016/S2213-8587(13)70211-0)

3 Rossi R, Tauchmanova L, Luciano A, Di Martino M, Battista C, Del Viscovo L, Nuzzo V \& Lombardi G. Subclinical Cushing's syndrome in patients with adrenal incidentaloma: clinical and biochemical features. Journal of Clinical Endocrinology and Metabolism $2000 \mathbf{8 5}$ 1440-1448. (https://doi.org/10.1210/jcem.85.4.6515)

4 Di Dalmazi G, Vicennati V, Rinaldi E, Morselli-Labate AM, Giampalma E, Mosconi C, Pagotto U \& Pasquali R. Progressively increased patterns of subclinical cortisol hypersecretion in adrenal incidentalomas differently predict major metabolic and cardiovascular outcomes: a large cross-sectional study. European Journal of Endocrinology 2012166 669-677. (https://doi.org/10.1530/EJE-11-1039)

5 Erbil Y, Ademoglu E, Ozbey N, Barbaros U, Yanik BT, Salmaslioglu A, Bozbora A \& Ozarmagan S. Evaluation of the cardiovascular risk in patients with subclinical Cushing syndrome before and after surgery. World Journal of Surgery 200630 1665-1671. (https://doi.org/10.1007/ s00268-005-0681-x)

6 Morelli V, Reimondo G, Giordano R, Della Casa S, Policola C, Palmieri S, Salcuni AS, Dolci A, Mendola M, Arosio M, et al. Longterm follow-up in adrenal incidentalomas: an Italian multicenter study. Journal of Clinical Endocrinology and Metabolism 201499 827-834. (https://doi.org/10.1210/jc.2013-3527)

7 Lopez D, Luque-Fernandez MA, Steele A, Adler GK, Turchin A \& Vaidya A. 'Nonfunctional' adrenal tumors and the risk for incident diabetes and cardiovascular outcomes: a cohort study. Annals of Internal Medicine 2016165 533-542. (https://doi.org/10.7326/M16-0547)

8 Bjorntorp P. Metabolic difference between visceral fat and subcutaneous abdominal fat. Diabetes and Metabolism 200026 (Supplement 3) 10-12.

9 Marin P, Darin N, Amemiya T, Andersson B, Jern S \& Bjorntorp P. Cortisol secretion in relation to body fat distribution in obese premenopausal women. Metabolism: Clinical and Experimental 1992 41 882-886. (https://doi.org/10.1016/0026-0495(92)90171-6)

10 Stolk RP, Lamberts SW, de Jong FH, Pols HA \& Grobbee DE. Gender differences in the associations between cortisol and insulin in healthy subjects. Journal of Endocrinology 1996149 313-318. (https:// doi.org/10.1677/joe.0.1490313)

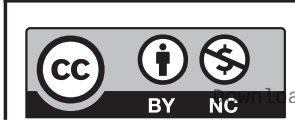

This work is licensed under a Creative Commons Attribution-NonCommercial 4.0 International License. ded from Bioscientifica.com at 04/26/2023 12:05:17AM 
11 Phillips DI, Barker DJ, Fall CH, Seckl JR, Whorwood CB, Wood PJ \& Walker BR. Elevated plasma cortisol concentrations: a link between low birth weight and the insulin resistance syndrome? Journal of Clinical Endocrinology and Metabolism 199883 757-760. (https://doi. org/10.1210/jcem.83.3.4634)

12 Filipovsky J, Ducimetiere P, Eschwege E, Richard JL, Rosselin G \& Claude JR. The relationship of blood pressure with glucose, insulin, heart rate, free fatty acids and plasma cortisol levels according to degree of obesity in middle-aged men. Journal of Hypertension 1996 14 229-235.

13 Litchfield WR, Hunt SC, Jeunemaitre X, Fisher ND, Hopkins PN, Williams RR, Corvol P \& Williams GH. Increased urinary free cortisol: a potential intermediate phenotype of essential hypertension. HYP.31.2.569)

14 Pasquali R, Cantobelli S, Casimirri F, Capelli M, Bortoluzzi L, Flamia R, Labate AM \& Barbara L. The hypothalamic-pituitaryadrenal axis in obese women with different patterns of body fat distribution. Journal of Clinical Endocrinology and Metabolism 199377 341-346. (https://doi.org/10.1210/jcem.77.2.8393881) Fardella C. Urinary free cortisol is not a biochemical marker of hypertension. American Journal of Hypertension 200720 459-465. (https://doi.org/10.1016/j.amjhyper.2006.09.017)

16 Praveen EP, Sahoo JP, Kulshreshtha B, Khurana ML, Gupta N, Dwivedi SN, Kumar G \& Ammini AC. Morning cortisol is lower in obese individuals with normal glucose tolerance. Diabetes, Metabolic Syndrome and Obesity: Targets and Therapy 20114 347-352. (https:// doi.org/10.2147/DMSO.S23915)

17 Abraham SB, Rubino D, Sinaii N, Ramsey S \& Nieman LK. Cortisol, obesity, and the metabolic syndrome: a cross-sectional study of obese subjects and review of the literature. Obesity 201321 E105-E117. (https://doi.org/10.1002/oby.20083)

18 Expert Panel on Detection, Evaluation, and Treatment of High Blood Cholesterol in Adults. Executive summary of the third report of the National Cholesterol Education Program (NCEP) expert panel on detection, evaluation, and treatment of high blood cholesterol in adults (Adult Treatment Panel III). JAMA 2001285 2486-2497. (https://doi.org/10.1001/jama.285.19.2486)

19 Tzoulaki I, Liberopoulos G \& Ioannidis JP. Assessment of claims of improved prediction beyond the Framingham risk score. JAMA 2009 302 2345-2352. (https://doi.org/10.1001/jama.2009.1757)

20 Kathiresan S \& Srivastava D. Genetics of human cardiovascular disease. Cell 2012148 1242-1257. (https://doi.org/10.1016/j.cell.2012.03.001)

21 Baudrand R, Goodarzi MO, Vaidya A, Underwood PC, Williams JS, Jeunemaitre X, Hopkins PN, Brown N, Raby BA, Lasky-Su J, et al. A prevalent caveolin-1 gene variant is associated with the metabolic syndrome in Caucasians and Hispanics. Metabolism: Clinical and Experimental 201564 1674-1681. (https://doi.org/10.1016/j. metabol.2015.09.005)

22 Pojoga LH, Underwood PC, Goodarzi MO, Williams JS, Adler GK, Jeunemaitre X, Hopkins PN, Raby BA, Lasky-Su J, Sun B, et al. Variants of the caveolin-1 gene: a translational investigation linking insulin resistance and hypertension. Journal of Clinical Endocrinology and Metabolism 201196 E1288-E1292. (https://doi.org/10.1210/ jc.2010-2738)

23 Peffer ME, Chandran UR, Luthra S, Volonte D, Galbiati F, Garabedian MJ, Monaghan AP \& DeFranco DB. Caveolin-1 regulates genomic action of the glucocorticoid receptor in neural stem cells. Molecular and Cellular Biology 201434 2611-2623. (https://doi. org/10.1128/MCB.01121-13) Hypertension 199831 569-574. (https://doi.org/10.1161/01.

15 Krall P, Carvajal C, Ortiz E, Munoz C, Garrido JL, Mosso L \&

24 Matthews L, Berry A, Ohanian V, Ohanian J, Garside H \& Ray D. Caveolin mediates rapid glucocorticoid effects and couples glucocorticoid action to the antiproliferative program. Molecular Endocrinology 200822 1320-1330. (https://doi.org/10.1210/me.20070154)

25 Vaidya A, Forman JP, Underwood PC, Hopkins PN, Williams GH, Pojoga LH \& Williams JS. The influence of body mass index and renin-angiotensin-aldosterone system activity on the relationship between 25-hydroxyvitamin D and adiponectin in Caucasian men. European Journal of Endocrinology 2011164 995-1002. (https://doi. org/10.1530/EJE-11-0025)

26 Vaidya A, Underwood PC, Hopkins PN, Jeunemaitre X, Ferri C, Williams GH \& Adler GK. Abnormal aldosterone physiology and cardiometabolic risk factors. Hypertension 201361 886-893. (https:// doi.org/10.1161/HYPERTENSIONAHA.111.00662)

27 Garza AE, Rariy CM, Sun B, Williams J, Lasky-Su J, Baudrand R, Yao T, Moize B, Hafiz WM, Romero JR, et al. Variants in striatin gene are associated with salt-sensitive blood pressure in mice and humans. Hypertension 201565 211-217. (https://doi.org/10.1161/ HYPERTENSIONAHA.114.04233)

28 Baudrand R, Gupta N, Garza AE, Vaidya A, Leopold JA, Hopkins PN, Jeunemaitre X, Ferri C, Romero JR, Williams J, et al. Caveolin 1 modulates aldosterone-mediated pathways of glucose and lipid homeostasis. Journal of the American Heart Association 20165 (https://doi.org/10.1161/JAHA.116.003845)

29 Gatti R, Antonelli G, Prearo M, Spinella P, Cappellin E \& De Palo EF. Cortisol assays and diagnostic laboratory procedures in human biological fluids. Clinical Biochemistry 200942 1205-1217. (https:// doi.org/10.1016/j.clinbiochem.2009.04.011)

30 Chamarthi B, Kolatkar NS, Hunt SC, Williams JS, Seely EW, Brown NJ, Murphey LJ, Jeunemaitre X \& Williams GH. Urinary free cortisol: an intermediate phenotype and a potential genetic marker for a salt-resistant subset of essential hypertension. Journal of Clinical Endocrinology and Metabolism 200792 1340-1346. (https://doi. org/10.1210/jc.2006-2093)

31 Kosachunhanun N, Hunt SC, Hopkins PN, Williams RR, Jeunemaitre X, Corvol P, Ferri C, Mortensen RM, Hollenberg NK \& Williams GH. Genetic determinants of nonmodulating hypertension. Hypertension 200342 901-908. (https://doi.org/10.1161/01. HYP.0000095615.83724.82)

32 Rao AD, Sun B, Saxena A, Hopkins PN, Jeunemaitre X, Brown NJ, Adler GK \& Williams JS. Polymorphisms in the serum- and glucocorticoid-inducible kinase 1 gene are associated with blood pressure and renin response to dietary salt intake. Journal of Human Hypertension 201327 176-180. (https://doi.org/10.1038/jhh.2012.22)

33 Wright KD, Hickman R \& Laudenslager ML. Hair cortisol analysis: a promising biomarker of HPA activation in older adults. Gerontologist 201555 (Supplement 1) S140-S145. (https://doi.org/10.1093/geront/ gnu174)

34 Hodes A, Lodish MB, Tirosh A, Meyer J, Belyavskaya E, Lyssikatos C, Rosenberg K, Demidowich A, Swan J, Jonas N, et al. Hair cortisol in the evaluation of Cushing syndrome. Endocrine 201756 164-174. (https://doi.org/10.1007/s12020-017-1231-7)

35 Walker BR, Best R, Shackleton CH, Padfield PL \& Edwards CR. Increased vasoconstrictor sensitivity to glucocorticoids in essential hypertension. Hypertension 199627 190-196.

36 Sudhir K, Jennings GL, Esler MD, Korner PI, Blombery PA, Lambert GW, Scoggins B \& Whitworth JA. Hydrocortisone-induced hypertension in humans: pressor responsiveness and sympathetic function. Hypertension 198913 416-421. (https://doi.org/10.1161/01. HYP.13.5.416)

Received in final form 10 April 2019

Accepted 23 April 2019

Accepted Preprint published online 23 April 2019
This work is licensed under a Creative Commons Attribution-NonCommercial 4.0 International License. ded from Bioscientifica.com at 04/26/2023 12:05:17AM 